\title{
EVOLUTION OF STARS DECREASING IN MASS
}

\author{
A. G. MASSEVITCH \\ Sternberg Astronomical Institute, Moscow
}

By a "steady" evolution of a star is understood such a change of its main parameters in the flow of time, which occurs in the range of an assumed equilibrium model and is caused by regular interior processes. So, for instance, the gradual conversion of hydrogen into helium in the convective core of a star, whose substance is not mixed fully, leads to a continuous change of its luminosity, radius, $T_{c}$ and $\rho_{c}$, convective core dimensions. However, all these changes occur all the time in the limits of the model's state of equilibrium, and the structure of the star (radiative envelope and convective core) remains unchanged until a certain limit is reached in the ratio $[I, 2,3]$.

$$
\frac{\mu \text { (core) }}{\mu \text { (envelope) }}=y_{\text {crit }}
$$

After the value $\mathrm{y}_{\text {crit., }}$ corresponding to the almost full exhaustion of hydrogen $\mathrm{X}$ in the convective core, is reached, further "steady" evolution becomes impossible, since for the further decrease of $\mathrm{X}$ in the range of the assumed model there are no equilibrium configurations. The subsequent development is already connected with the reconstruction of the star - with an unstable condition.

In reality the evolutionary track of a star is composed of a series of stages of "steady" evolutions of different duration, which are interrupted by intermittent changes (in the main of shorter duration). That is most convincingly confirmed by the Hertzsprung-Russell diagram.

It naturally is impossible to study intermittent changes characterizing unstable states of stars with the aid of ordinary equilibrium models. However, up to the present time there is no theory of unstable states of stars, which would be developed to any extent (except some interesting and promising, but so far only purely qualitative estimates).

At the same time, a thorough investigation of the "steady" evolution may supply some indirect information with respect to the nature of the next unstable stage, particularly, in relation to its boundary conditions. The theory of continuous evolution brings us closely to such a state of a star, with the beginning of which equilibrium is necessarily disturbed. The study of these states, based on physically reasonably constructed stellar models, is definitely of interest, not only to the theory of the interior structure of stars, but also for stellar cosmogony.

In the present report some possible tracks of the "steady" evolution of stars of continuously decreasing mass are treated.

2. EVOLUTION OF MASSIVE STARS -
THE BASIC DIFFICULTY IN THEOR Y

According to Vogt and Russell the equilibrium state of a star is uniquely determined by $\mu$ and $\mathfrak{O}$. Thermonuclear energy sources predetermine with sufficient certitude the change of $\mu$ with $t$, thus limiting the possible directions of evolution. In regard to $\mathfrak{N}$, generally speaking, 
there are two possibilities : $\mathfrak{N}=$ Constant and $\mathfrak{l} \neq$ Constant (for the solution of the problem there is no difference in principle between the decrease, or increase of mass). $\mathfrak{N}=$ Constant is the most evident and seemingly the simplest possibility, since it needs no additional hypotheses and for the majority of known stars it is not in contradiction with observations.

It is comparatively easy to calculate all possible paths of stellar evolution, and that has been done many times [2-6]. Especial successes in this direction were attained thanks to the works of Schwarzschild and his collaborators $[4,5]$, as a result of which the theory of evolution of stars of population II was developed. In particular, the Hertzsprung-Russell diagrams for globular clusters were explained, and a basic was also established for the theory of nucleogenesis in stars [7].

However, all calculations made up to now, which actually can be compared with observations, relate to stars of small mass, less than $2 \mathfrak{N} \odot$. The authors themselves [4] especially mention this limitation in their works. It is to be regretted that this is not always taken into account by those who use available results of such calculations.

All evolutionary hypotheses are confronted with serious difficulties, when attempts are made to explain the development of hot massive stars of the first part of the main sequence. One of these difficulties is sufficiently well known and consists in the fact that massive stars of early spectral types very lavishly exhaust their supply of nuclear fuel, due to which their possible stages of "steady" evolution are short in comparison with the age of the Galaxy. These stars quickly "pass out" of the main sequence, and the problem consists not so much in the explanation of the actual presence of such stars on the main sequence, in spite of this "passing out" (since it, seemingly, is now generally accepted that the process of star formation continues even now), as the need to know what the total aggregate of stars which have "passedout" gets converted into.

If we assume that the process of star formation is, in the main, uniform during the life of the Galaxy, then, as Salpeter [8] has shown, the total mass of stars of early spectral types that have "passed out" in 6.109 years from the main sequence (assuming that all of them evolved with constant mass) is comparable with the total mass of all stars that are now in the Galaxy, i.e., it is equal to Io $^{11} \mathfrak{N} \odot$.

The query then arises - where is that mass and in what state?

Lately a growing popularity is gained by the following evolutionary scheme : hot giant - rapid transition to a red giant loosing mass - rapid conversion to a white dwarf.

It must, however, be kept in mind, that at the same time the loss of more than 9/10 of the mass of the star must be "provided" in such time periods that the stages of conversion would practically not be observed (since they are not observed on the Russell diagram), i.e., at any rate, they should be considerably shorter than the life of such a star in the stage of "steady" evolution of a white or red giant. The lifetime on the main sequence is about $\sim \mathrm{IO}^{7}$ years for an $\mathrm{O}$ star and $\sim \mathrm{IO}^{8}$ years for a $\mathrm{B}$ star.

If we assume that the evolutionary stage, which proceeds a hundred times faster, is practically already inobservable, then the decrease in mass of the remnants of the former $\mathrm{O}$ and B stars must proceed with a velocity

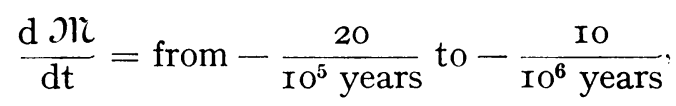

i.e., - $\mathrm{IO}^{22} \mathrm{~g} / \mathrm{sec}$. - $\mathrm{IO}^{21} \mathrm{~g} / \mathrm{sec}$, which by 5-4 orders exceeds the loss by radiation for such stars and by $4-3$ orders exceeds the rate of loss of mass by red supergiants, according to modern 
data (According to Deutch [9] for $\alpha \mathrm{Her}, \frac{\mathrm{d} \mathfrak{l} \mathcal{L}}{\mathrm{dt}}=-\mathrm{Io}^{18} \mathrm{~g} / \mathrm{sec}$.). It must also be noted that no calculations of evolutionary tracks were made at such an intensity of decrease of mass between the boundary stages red supergiant to white dwarf (and certainly no kind of mechanism for such outflow was considered). From the point of view of the theory of the interior structure of stars we are here confronted with very serious difficulties.

Thus, the theory of evolution at constant mass leads in stars of early spectral types of the main sequence to the necessity of introducing the assumption of an intense decrease of mass for these stars at later stages, so that such an evolution may be called a constant mass evolution only very loosely.

The second difficulty, which usually does not receive the attention it deserves, consists in the fact that the assumption of constancy of mass is in contradiction with the structure of the main sequence (its first part). Indeed, the first part of the main sequence is a homogeneous group of stars for which there practically exist functional relations : mass - luminosity and mass - radius.

These relations have the form [Io] :

$$
\begin{gathered}
\frac{\mathrm{L}}{\mathrm{L}_{\odot}}=\operatorname{I.I2}\left(\frac{\mathfrak{N}}{\mathfrak{M}_{\odot}}\right)^{3.9}, \\
\frac{\mathrm{R}}{\mathrm{R}_{\odot}}=\left(\frac{\mathfrak{M}}{\mathfrak{M}_{\odot}}\right)^{0.75} .
\end{gathered}
$$

That means that in the space $\mathrm{L}-\mathfrak{l} i-\mathrm{R}$ these stars form a linear sequence and that the mean molecular weight of the group under consideration is either constant, or is a continuous function of mass.

Let us compare empirical relations (I) and (2) with the two theoretical relations (L, $\mathfrak{n}, \mathrm{R}, \mu)$, which follow from the theory of the internal structure of stars and of stellar energy sources,

where $\mathrm{k}=\mathrm{k}_{\mathrm{o}} \rho^{\alpha} \mathrm{T}^{-\beta}$.

$$
\mathrm{L}=\text { Const. } \frac{\mu^{4+\beta}}{\mathrm{k}_{0}} \Re^{3+\beta-\alpha} \mathrm{R}^{2 \alpha-\beta},
$$

where $\varepsilon=\varepsilon_{0} \rho T^{\mathrm{n}}$.

$$
\mathrm{L}=\text { Const. } \mu^{\mathrm{n}} \times Z \mathfrak{l l}^{\mathrm{n}+2} \mathrm{R}^{-\mathrm{n}-3} \text {, }
$$

Any reasonable assumption with respect to $\alpha, \beta, \mathrm{n}$ will always give the following relation :

$$
\mu=\mu(\mathfrak{M}) \text {, }
$$

which indicates that $\mu$ changes continuously along the sequence. Many authors [I0, II, I2, I3] have more than once reached this result. For one and the same stellar model (even if the law of absorption along the sequence would be modified within reasonable limits) calculations always give a systematic change of the chemical composition along the sequence (The greater the mass of the star, the greater is $\mathrm{X}$ and the less $\mathrm{Y}$ ).

The sequence of homogeneous stars for a same $\mu$ has no similarity whatsoever with the actual main sequence and lies from $3^{\mathrm{m}}$ to $5^{\mathrm{m}}$ below it on the Russell diagram (see Fig. I).

Lately, (perhaps for the first time at the Rome I.A.U. Congress) the opinion has spread that this difficulty disappears as soon as we consider the evolution of unmixed stars. In that case the main sequence is no longer interpreted as a sequence of homologous stars, but is consider- 
ed as the totality of inhomogenous stars of the same age, but at different stages of evolution. The more massive is a star, the greater is its inhomogeneity, i.e., it belongs to a later evolutionary stage. In the entire sequence, star envelopes retain the same initial chemical composition;

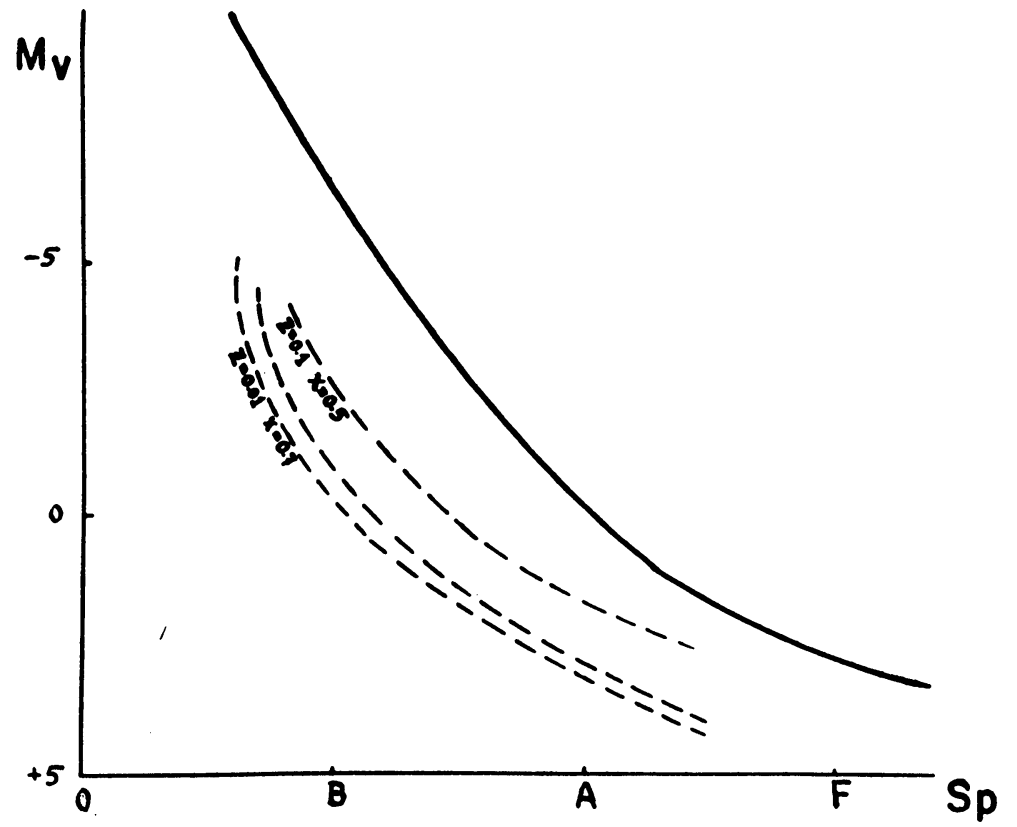

FIGURE I. Theoretical sequences of homogeneous stellar models on the HR diagram with $\mu=$ Const along the sequence.

The solid line represents the real main sequence according to [10]. the latter is different only in their central parts, depending upon the stage of evolution.

However, detailed calculations show that the introduction of inhomogeneity also does not solve the problem for massive stars. The results of calculations of the evolution of inhomogeneous stars from ro $\mathfrak{R}_{\odot}, 5 \mathfrak{r}_{\odot}$ and $2.5 \mathfrak{N r}_{\odot}$ by Kushwaha [14] are shown in Fig. 2.

In these calculations inhomogeneity was considered fully : the dimensions and mass of convective cores are different, each star also has various transient zones with a continuously changing $\mu$.

On Fig. 2 are shown: the zero-age sequence, the line $t=4 \times 10^{7}$ years and the line $\mathrm{t}=\mathrm{I} .5 \times 10^{8}$ years.

Not a single one of these lines coincides with the main sequence. The calculation of inhomogeneity only somewhat lessened the divergence between the actual and theoretical main sequence; however, no change of the initial $\mu$ within reasonable limits can eliminate it altogether.

In order that both curves coincide it is necessary, as has been done before, to assume that $\mu$ is changing along the sequence.

In the range of the stellar evolutionary theory at constant mass the actual main sequence for early type stars can be explained only by the assumption that all these stars have already been formed with a different chemical composition, so that $\mu=\mu(\mathcal{M})$. In essence, that only means that the difficulty has been shifted to an earlier stage.

\section{E V OL U T O N W I TH DECREA S N G M A S}

Both of the difficulties for massive stars described above disappear, if we introduce into the evolutionary theory the conception of the continuous decrease of the mass of stars.

The first part of the main sequence is then interpreted as the result of the evolution of stars of the same structure which have been formed at different times and in different parts of the Galaxy. 
Assuming that due to corpuscular radiation a star loses mass continuously (by a law, which so far is unknown to us), but in such a manner that it does not leave the limits of the given group of star, then its evolutionary track on the HR diagram may be calculated, utilizing theoretical relations (3) and (4).

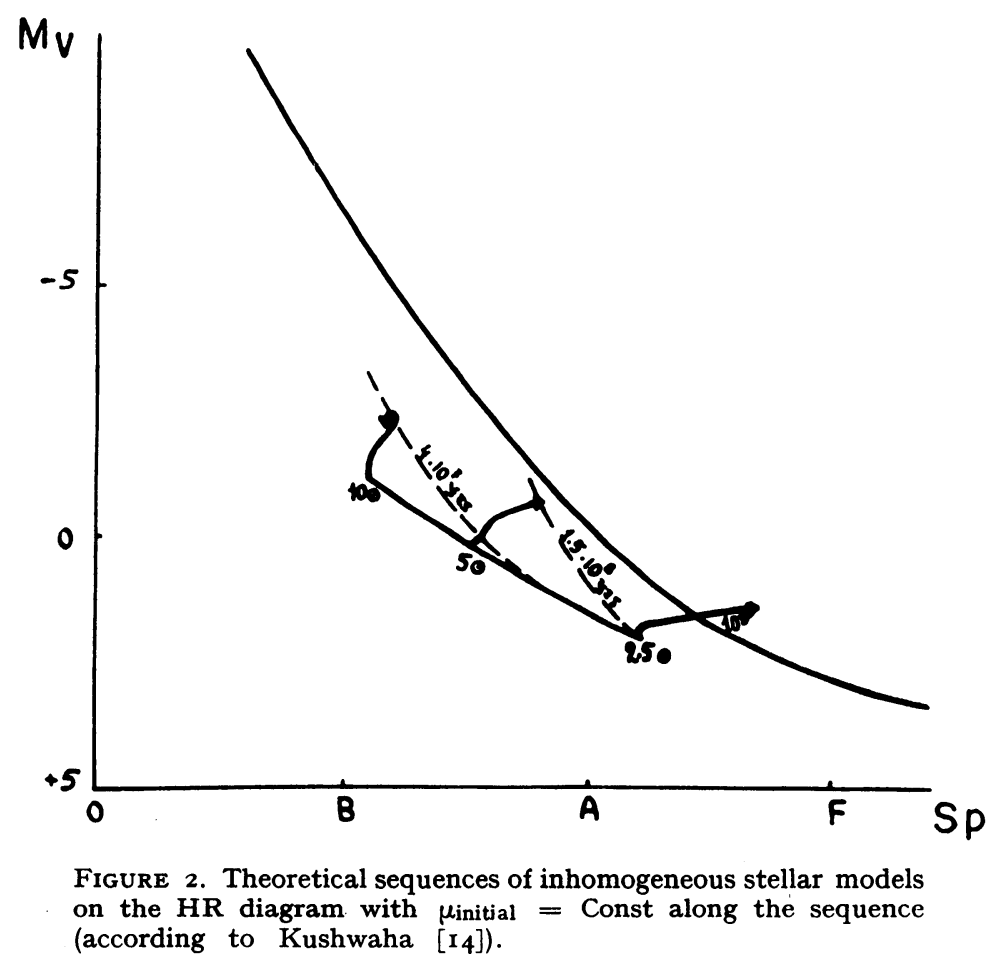

In order that the sequence of stars built in agreement with (3) and (4) would actually be the observed main sequence, it is, as has been shown above, necessary that along with (3) and (4) the condition be fulfilled that

$$
\mu=\mu(\mathfrak{\jmath}) \text {, }
$$

which is determined by the empirical relations $L \_\Re$ and $R \_\Re$, and which for stars of the first part of the main sequence takes on the form

$$
\frac{\mu^{4+\beta}}{\mathrm{k}_{0}}=\text { Const. } \mathfrak{\Re - 1 . 2 5 \alpha - 0 . 2 5 \beta + 0 . 9} \text {. }
$$

For stars of the second part of the main sequence, for which there are other relations $\mathrm{L}=\mathrm{L}(\mathfrak{M})$ and $\mathrm{R}=\mathrm{R}(\mathfrak{N})$, the relation $\mu(\mathfrak{N})$ will also be different. It will also be different for giants and supergiants, for which the mass - luminosity and mass - radius relations will differ from the relations for the main sequence.

We have treated the relation $\mu(\mathcal{N})$ for different sequences in detail in [Io].

Adding to equations (3) and (4) various relations, $\mu=\mu(\mathcal{2})$, we single out from the entire totality of stars, which a theoretical model of a gasous star with a convective core and 
thermonuclear source of energy may describe, the stars of the sequence that is of interest to us. In particular, by the addition of relation (5) to equations (3) and (4), or, which is the same, by the addition of (I) and (2) to (3) and (4) we single out the first part of the main sequence.

In order to substantiate the hypothesis advanced above, that this sequence characterizes the changes of a star with time, i.e. that it may be interpreted as evolutionary, it is necessary to show that the change of $\mu$ along the sequence given by (5) may be explained by the conversion

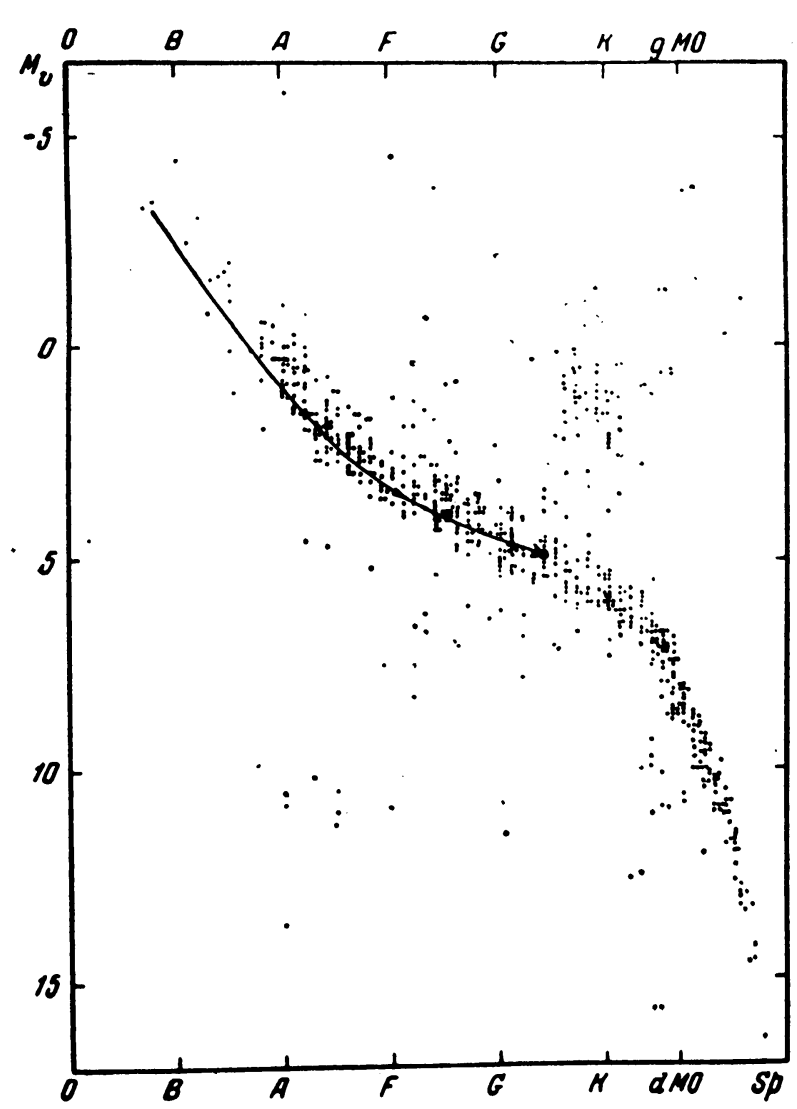

FIGURE 3. The theoretical main sequence for $Z=Z_{\odot}=$ 0.04 on the HR diagram for evolution with decreasing mass. of hydrogen into helium, as a result of nuclear reactions in the interior of a star, the mass of which continuously decreases, due to corpuscular radiation, while at the same time the abundance of heavy elements $Z$ does not change. Solving equations (3) and (4) together with the equation (I) (the mass-luminosity relation) and assuming that $Z=$ Const. (which will really mean that the change of $\mu$ is conditioned only by the conversion of $\mathrm{X}$ into $\mathrm{Y}$ ), we must obtain relation (2) - the mass - radius relation of stars of the first part of the main sequence.

In other words, if the proposed evolutionary interpretation of the main sequence is true, then the joint solution of (3), (4) and (I) for $Z=$ Const. must theoretically produce the main sequence on the HR diagram.

It must be noted, that, by assuming $Z=$ Const., we put very rigid restrictions upon the possible changes of $\mathrm{L}, \mathfrak{N}, \mathrm{R}$, since the average molecular weight of stars can be changed only in a very limited interval of values from $I / 2$ to $\sim 2$, while $X$ and $Z$ arevery sensitive to changes of $\mathfrak{R}$ and $R$.

As has been shown in $[\mathrm{I}, 6, \mathrm{II}]$, as a result of such calculations we actually arrive at the shape of the main sequence (see Fig. 3).

The analysis of calculations made for other $\mu(\mathcal{N} i)$, or similarly for other $\gamma$ (mass exponents in equation (I)), shows [I5], that of all possible values of $\gamma$ only for $\gamma=3.9$ does the theoretical curve correspond to the sequence of stars, for which simultaneously the two-dimensional relations mass - luminosity and mass - radius exist.

The value $\gamma=3.9$ just exactly characterizes the first part of the main sequence.

The theoretical evolutionary curve $Z=$ Const., for some initial chemical composition, symbolically designated by $Z_{\odot}\left(Z_{\odot}=0.04\right)$ is drawn on Fig. 3 .

The curve reproduces well the shape of the first part of the mair sequence. The totality of such evolutionary curves for various values of $\frac{1}{2} Z_{\odot}<Z<2 Z_{\odot}$ may give the actually observed main sequence with its real dispersion along $M_{v}$, which cannot be reduced to errors 
of observation. That, in its turn, indicates, that the main sequence is interpreted as the result of the evolution of stars formed at different times and in different parts of the Galaxy (different $Z$ ).

Hence, the upper part of the main sequence is the result of the evolution of stars formed in the main as stars of earliest spectral classes, from whose surfases gas flows off, which leads to the decrease of mass. Simultaneously, in the interiors of these stars, as a result of nuclear reactions, hydrogen is converted into helium, while in the meanwhile the abundance of heavy elements $Z$ remains unchanged. This conditions the change of the chemical composition of stars with time, i.e., the increase of $\mu$ with the decrease of $\mathfrak{N}$ (it is assumed that at early stages mixing is complete).

The evolution of a homogeneous star with decreasing mass, permits us to explain the changes of $\mu$, of the velocity of rotation, of age and other characteristics of stars along the main sequence. It explains the actual dispersion of the main sequence, peculiarities of the HR diagrams for open clusters, and yields the value of the limiting mass of a star of the main sequence corresponding to the earliest spectral class $\mathrm{O}_{5}[6, \mathrm{II}, \mathrm{I} 5]$.

The greatest merit of this hypothesis is the "prolongation" of the age of massive stars. As calculations have shown $[6, \mathrm{II}, \mathrm{I} 6]$, the time in which an originally massive hot star passes consecutively through stages corresponding to later and later spectral classes, at the same time remaining in the limits of the main sequence, constitutes several milliards of years. Such evolution stops when mixing ceases in a star, after which the star begins to deviate from the main sequence. Various values of mass (from $\mathfrak{M}=3 \mathfrak{K}_{\odot}$ and less) give the arrival of this stage of development for various stars. Further development may proceed either along the path of constant-mass evolution (here the star deviates from the main sequence almost vertically upwards), or along the diminishing-mass evolutionary track, but without mixing (here for some time the star is still displaced downwards, along the main sequence, simul-

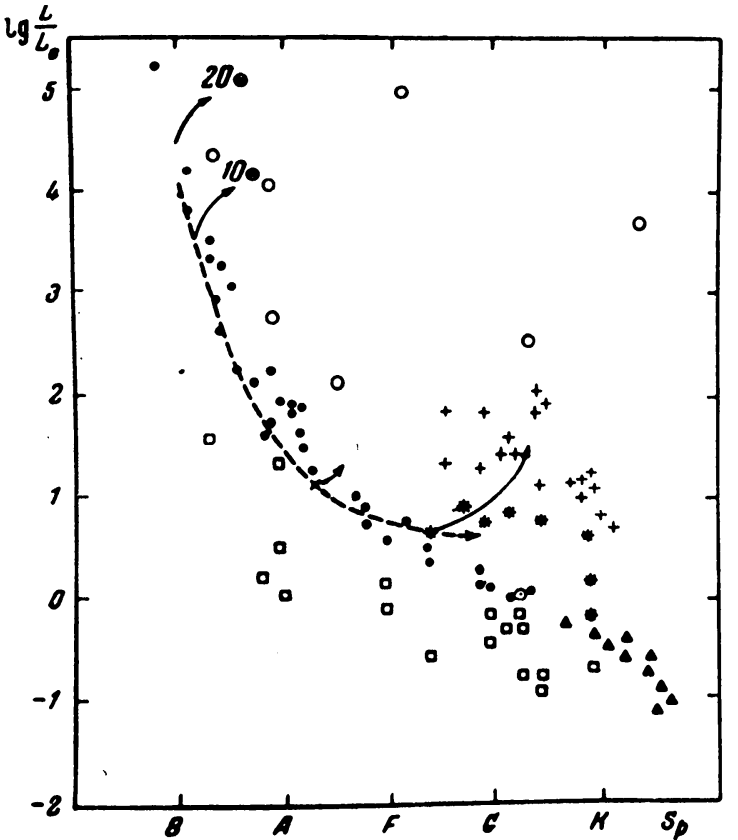

Fig. 4. Possible evolutionary path of a hot early-type star $(\mathrm{BI})$ consisting of several parts

$\longrightarrow-\longrightarrow$ evolution with decreasing mass (till + with mixing; after + - without mixing).

evolution with constant mass (short arrow- " steady" evolution, long arrow-evolution with contracting core according to M. Schwarzschild).

- stars of the first part of the main sequence.

$\Delta$ stars of the second part of the main sequence.

subdwarfs.

* subgiants.

+ giants.

O supergiants. Data for real stars were taken from [10]. taneously being deviated upwards $[6, \mathrm{II}]$. However, all these stages are of short duration as compared with the time during which a star moves near to or on the main sequence [I6].

The evolution of a hot star of the main sequence is shown on Fig. -4 - an evolution consisting of a few stages. A star of spectral class B I $\left(\mathfrak{k}=8 \mathfrak{i} \boldsymbol{i}_{\odot}\right)$ evolves along the main sequence, losing mass and consecutively passing through stages of a star of later and later 
spectral classes, until full mixing within it ceases. In our example it corresponds to the value $\mathfrak{K}={ }_{2} \mathfrak{K}_{\odot}$. This stage is designated on the diagram by a cross. On Fig. 4 are also shown the further stages of evolution without mixing. For each stage its duration is indicated. The greatest $\left(\sim 4 \times 10^{9}\right.$ years) is the first stage, in which the star is moving nearly on the main sequence. In this manner, from the proposed evolutionary interpretation of the main sequence it follows that the vast majority of stars of the main sequence spend a considerable span of their lives on it, and that the number of stars, which have "passed out" of the main sequence during the life of the Galaxy, is relatively small.

If we assume that stars are being formed continuously as massive stars of early spectral classes, after which the vast majority of these newly generated stars evolves along the main sequence, then it may be shown [I6] that for the derivation of the observed luminosity function of main-sequence stars it is necessary that the number of newly formed stars should correspond to $5 \times 10^{8}$ stars in $10^{10}$ years. This number is in good agreement with the estimation made by V. A. Ambarzumjan for the number of stars formed in $\mathrm{O}$ - associations for a period of $10^{10}$ years $\left(10^{8}-10^{9}\right)$ stars. At the same time a considerable fraction of the mass of these stars is dispersed in interstellar space. As the analysis of the luminosity function [16] shows, about Io to $15 \%$ of the newly formed stars do not evolve along the sequence, leaving it to the right and upwards, when there is no mixing ("turn off" of main sequences in open clusters), or to the right and downwards, when mixing continues (stars of the $\lambda$ Boo type [I7]).

Nothing was said so far about the law of the diminution of mass. It may be derived from the following considerations :

The change of hydrogen in a star is :

$$
\begin{gathered}
\frac{\mathrm{dX} \Re \tilde{L}}{\mathrm{dt}}=-\frac{\mathrm{L}}{\alpha}-(\text { ejection of mass per sec). X, } \\
\alpha=6.4 \cdot 10^{18} \mathrm{ergs} / \mathrm{g} .
\end{gathered}
$$

The full loss of mass by a star is :

$$
\frac{\mathrm{d} \mathfrak{N}}{\mathrm{dt}}=\left(\text { ejection of mass per sec) }-\frac{\mathrm{L}}{\mathrm{c}^{2}}\right. \text {. }
$$

From (7) and (8) is derived :

$$
\mathfrak{N} \frac{\mathrm{dX}}{\mathrm{dt}}=\frac{\mathrm{L}}{\alpha}+\frac{\mathrm{XL}}{\mathrm{c}^{2}}
$$

On the other hand,

$$
\frac{\mathrm{d} \mathfrak{R}}{\mathrm{dt}}=\frac{\mathrm{d} \mathfrak{d}}{\mathrm{dX}} \cdot \frac{\mathrm{dX}}{\mathrm{dt}}
$$

Substituting $\frac{d X}{d t}$ from (9) and ignoring the second term, we will get $\frac{d \mathfrak{l}}{d t}=-\frac{L}{\alpha} \frac{d \ln \mathfrak{d}}{d X}$; which is very similar to the law proposed by V. G. Fessenkov [I8]

$$
\frac{\mathrm{d} \mathscr{N}}{\mathrm{dt}}=-\mathrm{kL} \text {. }
$$

if we assume that $\mathrm{k}=\frac{1}{\alpha} \frac{\mathrm{d} \ln \Re \Re}{\mathrm{dX}}$, i.e., $\mathrm{k}=\mathrm{k}(\mathrm{X})$.

$\mathrm{k}$ is slowly changing with $\mathrm{X}$. As calculations for $\Delta \mathrm{X}=0.5$ show, $\mathrm{k}$ increases one-anda-half times. 
For a star of type BO, whose evolution is shown on Fig. 4, the mean rate of the diminution of mass corresponds to

$$
\frac{\mathrm{d} \Re}{\mathrm{dt}}=-3 \cdot \mathrm{IO}^{17} \mathrm{~g} / \mathrm{sec},
$$

i.e., it is four orders of magnitude less than the rate of decrease of mass of such a star, if as the result of constant-mass evolution it should be converted into a red giant (see section 2).

$\frac{\mathrm{d} \Re \mathrm{K}}{\mathrm{dt}}=-3 \cdot 10^{17} \mathrm{~g} / \mathrm{sec}$ is comparable with the data on diminution of mass in some B-stars treated by various authors: $\mathrm{P}$ Cyg $\sim \mathrm{IO}^{21} \mathrm{~g} / \mathrm{sec}\left(\right.$ Pagel), Pleione $\sim \mathrm{IO}^{17}-\mathrm{IO}^{20} \mathrm{~g} / \mathrm{sec}$. (Underhill), II Cam $\sim \mathrm{IO}^{18} \mathrm{~g} / \mathrm{sec}$. (Burbidges) and others. A summary of all such data is presented in [I9].

All the above mentioned, of course, tells us nothing about the ejection mechanism, proper, which so far remains altogether indefinite, which is the main defect of the proposed conception. We must say that with respect to constant-mass evolution that this holds good to a still greater extent.

Let us treat the Hyades cluster diagram from the point of view of evolution with decreasing mass. This diagram is shown in Fig. 5, in accordance with Eggen's data; the theoretical curve $Z=0.87 Z_{\odot}$ represents the cluster main sequence best of all. The evolution of the main mass of stars proceeds along this curve. The deviation of a part of bright stars to the right and upwards is satisfactorily explained by the evolution without mixing. Here also are

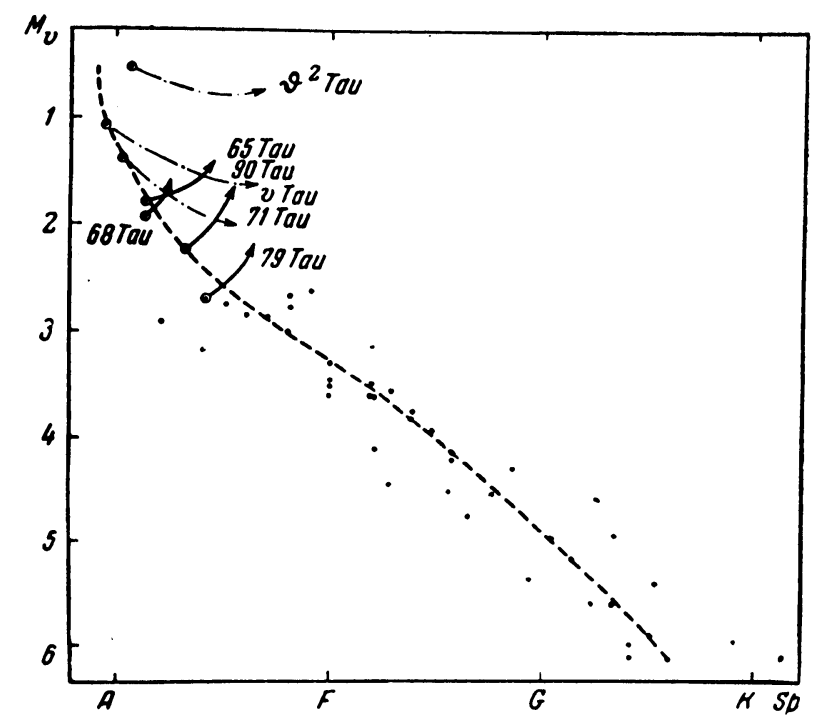

FIGURE 5. Interpretation of the HR Diagram for the Hyades.

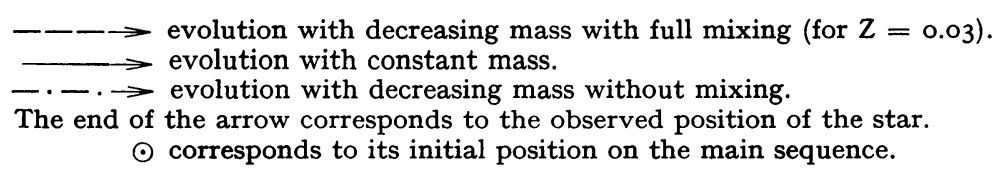

drawn their evolutionary curves, and their initial positions on the main sequence are indicated by open circles. The diminishing-mass evolution is indicated by dashed lines with arrows. For these three stars $V_{r} \sin i$ is in excess of $100 \mathrm{~km} / \mathrm{sec}$. Solid lines with arrows indicate constant- 
mass evolution; ( $\mathrm{V}_{\mathrm{r}} \operatorname{sini}$ is less than $100 \mathrm{~km} / \mathrm{sec}$ for all of these stars). Most of the stars with relatively great rotation are moving on the main sequence. The time required for the brightest star of the cluster $\theta^{2}$ Tau to move off the mean line of the initial main sequence to the position occupied by it now corresponds to $3 \times 10^{8}$ years. For a star of the same luminosity moving along the main sequence the time required for its conversion into the brightest star, which is still on the main sequence ( $79 \mathrm{Tau}$ ) also equals $4 \times 10^{8}$ years. Evidently, $\sim 3 \times 10^{8}$ years is the minimum possible life of the investigated cluster. Analogous calculations have been made for a series of other clusters and associations [II, I6].

REFERE N C E

[I] A Massevitch, L. Tulenkova, V. Matveeva, A. J. USSR 28, No. 6, I95I; Publ. Inst. Astr. and Physics, Acad. Sci. Kaz. SSR, I., I955.

[2] V. Sorokin and A. Massevitch, A. J., USSR 28, No. I, I95 I.

[3] P. Ledoux, Mem. Soc. Roy. Sc. Liège, 14, 1954.

[4] M. Schwarzschild and A. Sandage, Ap. J. 116, 463, 1952; M. Schwarzschild and F. Hoyle, Ap. J. Suppl. I3.

[5] A. Sandage, Mem. Soc. Roy. Sc. Liège 14, 254, r954.

[6] A. Massevitch, A. J. USSR 28, No. 5, 1951; 30, No. 4, 1953; Stern. Comm. No. 99, 1, 1956.

[7] M. Burbidge, G. Burbidge, W. Fowler, Ap. J. Suppl. I 7, 2, I 955.

[8] E. E. Salpeter, Ap. J. 121, I6I, I955.

[9] A. Deutsch Ap. J. 123, $210,1956$.

[10] P. Parenago and A. Massevitch, Stern. Publ. 20, I95I; A. J. USSR 27, No. 3, 4, I950.

[II] A. Massevitch, Mem. Soc. Roy. Sc. Liège 14, 244, I954.

[12] A. B. Severny, A. J. USSR 31, 362, I954.

[13] J. C. Pecker and E. Schatzman, Ann. d. Aph. 10, I81, 1947.

[14] Kushwaha, Ap. J. 121, No. 3, I955.

[15] G. Ruben and A. Massevitch, A. J. USSR 34, 724, 1957.

[I6] A. Massevitch, A. J. USSR 33, 216, 1956.

[17] A. Massevitch, A. J. USSR 35, 292, 1958.

[I8] V. Fessenkov, A. J. USSR 27, No. 2, I 949.

[19] M. E. Burbidge and G. Burbidge, Evolution of Stars, Handb. f. Ph. 51, I 958.

\section{Discussion}

Bondi : Mme. Massevitch's talk showed that the decision between constant-mass and decreasing-mass evolution depends on an exact comparison of theoretical and observed HR diagrams. A re-determination of the bolometric correction is therefore urgently required.

Massevitch : I quite agree that the most important thing we badly need is a re-determination of the bolometric corrections for early-type stars.

Savedoff : (I) I don't understand why Kushwaha and other Princeton people find reasonable fits to the main-sequence while you do not. Where does your data differ?

(2) Kushwaha's calculations for mass ro $\odot$ need revision (now underway at Rochester), since radiation pressure was neglected in the.cores. Detailed calculations now show that the mass of the convective core increases.

Massevitch : (I) I think the difference is due to the bolometric corrections. I remember that Kushwaha mentions a discrepancy between his initial main-sequence and the real one. He does not give the diagram there.

(2) I am not sure if this will not increase the discrepancy even more. 